\title{
Neutral atomic hydrogen absorption and the Galactic Center Excess
}

\author{
Martin Pohl, ${ }^{a, b}$ Phaedra Coleman, ${ }^{c}$ Chris Gordon ${ }^{c, *}$ and Oscar Macias ${ }^{d, e}$ \\ ${ }^{a}$ University of Potsdam, \\ Institute of Physics and Astronomy, D-14476 Potsdam, Germany \\ ${ }^{b} \mathrm{DESY}$, \\ D-15738 Zeuthen, Germany \\ ${ }^{c}$ School of Physical and Chemical Sciences, \\ University of Canterbury, Christchurch, New Zealand \\ ${ }^{d}$ Kavli Institute for the Physics and Mathematics of the Universe, \\ University of Tokyo, Kashiwa, Chiba 277-8583, Japan \\ ${ }^{e}$ GRAPPA Institute, \\ University of Amsterdam, 1098 XH Amsterdam, The Netherlands \\ E-mail: pohlmadq@gmail.com, bjc174@uclive.ac.nz, \\ chris.gordon@canterbury.ac.nz, oscar.maciasramirez@gmail.com
}

\begin{abstract}
An excess of gamma rays has been observed in the Galactic center region by Fermi-LAT. In order to characterise these gamma rays accurate estimates of the diffuse galactic background are needed. We improve the determination of the component of the diffuse galactic background caused by cosmic rays colliding with neutral atomic hydrogen $(\mathrm{HI})$. We account for both line and continuum emission in the radiation transport. This allows the modelling of negative line intensity and traces gas both in emission and absorption. We find Fermi-LAT templates generated from such a model provide an improved fit in comparison to templates generated from previous models which do not account for the continuum component. But this does not change the conclusion that the unresolved population of millisecond pulsars model of the GCE provides a better fit than the self-annihilating dark matter model of the GCE.
\end{abstract}

$37^{\text {th }}$ International Cosmic Ray Conference (ICRC 2021)

July 12th-23rd, 2021

Online - Berlin, Germany

\footnotetext{
${ }^{*}$ Presenter
} 


\section{Introduction}

Pohl et al. [11] used a gas-flow model based on the smoothed-particle hydrodynamics simulation described in Bissantz et al. [3] to deconvolve CO data. They employed an iterative method to successively reduce signal in the line spectrum and place it at the eight best-matching distance intervals, until there is only noise left. In Macias et al. [8] an analogous deconvolution of $\mathrm{HI}$ data was found to provide a better fit to the diffuse gamma-ray emission from the Galactic-center region than do the gas maps of the standard Fermi-LAT data analysis pipeline ${ }^{1}$ [see also 7]. The absorption correction was minimal and involved only self-absorption with constant excitation temperature $T_{\mathrm{exc}}=170 \mathrm{~K}$. Continuum emission was ignored, which means weak positive signal was deemed optically thin and negative signal had to be disregarded. In the Galactic-center region these simplifications lead to a potentially significant underestimation of the mass of atomic gas, and hence a deficit in the predicted diffuse gamma-ray emission and an artificial indication for new emission components.

In this paper we present an advanced model of atomic gas in the Galaxy and apply it to the analysis of gamma-ray emission from the Galactic center. We account for both line and continuum emission in the radiation transport, which allows the modelling of negative line intensity and traces gas in both emission and absorption.

\section{Method}

\subsection{Radiation transport}

The radiation transport equation for the intensity, $I$, along a line of sight, $s$, reads

$$
\frac{d I}{d s}=j_{c}+j_{l}-\alpha_{l} I
$$

where we allow for continuum emission with coefficient $j_{c}$, line emission, $j_{l}$, and absorption, $\alpha_{l}$. Continuum absorption is ignored, because the brightness temperature is always very much smaller than the excitation temperature for free-free emission, about $10^{4} \mathrm{~K}$. In our model the line of sight is binned, and the radiation coefficients are assumed to be constant within a bin. At the front of each bin of length $\Delta s$, corresponding to an optical depth $\tau=\Delta s \alpha_{l}$, we find for $\tau>0$

$$
I(\Delta s)=I_{0} \exp (-\tau)+\frac{j_{c}+j_{l}}{\alpha_{l}}[1-\exp (-\tau)]
$$

where $I_{0}$ is the intensity at the rear boundary.

The change of the intensity across the bin is given by

$$
\begin{aligned}
\Delta I & =I(\Delta s)-I_{0} \\
& =\left(\frac{j_{c}+j_{l}}{\alpha_{l}}-I_{0}\right)[1-\exp (-\tau)] \\
& =\left(\frac{j_{c} \Delta s+j_{l} \Delta s}{\tau}-I_{0}\right)[1-\exp (-\tau)]
\end{aligned}
$$

\footnotetext{
1https://fermi.gsfc.nasa.gov/ssc/data/access/lat/BackgroundModels.html
} 
We are working in the low frequency limit where the brightness temperature $T_{B}$ is proportional to the intensity. So the increment in $T_{B}$ is

$$
\Delta T_{B}=\left(\frac{\Delta T_{c}+\Delta T_{l}}{\tau}-T_{0}\right)[1-\exp (-\tau)],
$$

where $T_{0}$ is the brightness temperature at the rear boundary and $\Delta T_{c} \propto j_{c} \Delta s$ is the increment in continuum brightness temperature across the bin. The increment in the line brightness temperature is given by $\Delta T_{l}=\tau T_{\mathrm{exc}}$ where $T_{\mathrm{exc}} \propto j_{l} / \alpha_{l}$ is the excitation temperature of the atomic-hydrogen gas (see for example section 7.4.1 of [6]).

For each velocity bin and line of sight, we successively apply eq. 4 to find the radiation temperature at Earth, from which we subtract the continuum temperature to obtain the predicted line brightness temperature

$$
T_{l}=-T_{c}+\sum_{i} \Delta T_{B}\left(s_{i}\right)
$$

Matching those spectra to the observed line spectra will yield $\Delta T_{l}$ for each distance bin. The corresponding contribution to the column density of gas, $N_{H}$, is

$$
\Delta N_{H}=\left(1.8 \cdot 10^{18} \mathrm{~s} \mathrm{~K}^{-1} \mathrm{~cm}^{-2} \mathrm{~km}^{-1}\right) \Delta v \Delta T_{l},
$$

where $\Delta v$ is the bin width in velocity space.

\subsection{Continuum Modelling}

We use the CHIPASS ${ }^{2}$ [4] and Stockert ${ }^{3}[13,14]$ continuum datasets. The CHIPASS data was reprojected to match the Stockert data. Missing regions in the CHIPASS data are patched with the Stockert data. We then fit a model of the continuum emission consisting of three Gaussian disk components,

$$
\Delta T_{c} / \Delta s=\sum_{i=1}^{3} a_{i} \exp \left[-\frac{1}{2}\left(\frac{r^{2}}{\sigma_{r, i}^{2}}+\frac{z^{2}}{\sigma_{z, i}^{2}}\right)\right]
$$

where $a_{i}, \sigma_{r, i}$, and $\sigma_{z, i}$ where the fitted model parameters. The model was fitted in in Galactic centered cylindrical coordinates with radius $r$ and height above the Galactic plane $z$. On a ROI of $|l|<60^{\circ},|b|<25^{\circ}$, we fit one disk, then fix that and fit a second one. We then add a third component initialised to around the GC intense region's extent, which converged for that component. Then we free all parameters to fit the three components simultaneously using least-squares optimisation. The best fit parameters are shown in Table 1.

Using this three-Gaussians model, we created an instance of the model on the grid of the gas-deconvolution cube, and renormalised it to the observed continuum temperature for each line of sight, so the signal in the bins sums up to the observed continuum brightness. As the model is just a simple three-component model, the continuum cube has a few stark point-source-like components when renormalised, which manifest themselves as bright streaks through slices of the cube. The Galactic center is a hotspot in continuum brightness with $T_{c} \gtrsim 500 \mathrm{~K}$. Figure 1 displays the distribution of continuum emissivity per distance bin which likewise has a sharp peak at the Galactic center.

${ }^{2}$ https://lambda.gsfc.nasa.gov/product/foreground/fg_stockert_villa_info.cfm

${ }^{3}$ https://lambda.gsfc.nasa.gov/product/foreground/fg_chipass_info.cfm 
Table 1: Best fit parameter values for the three-disk continuum model.

\begin{tabular}{c|c|c|c}
\hline \hline Parameter & Amplitude $(a)$ & Radial standard deviation $\left(\sigma_{r}\right)$ & Vertical standard deviation $\left(\sigma_{z}\right)$ \\
\hline Units & $\mathrm{K} / \mathrm{kpc}$ & $\mathrm{kpc}$ & $\mathrm{kpc}$ \\
\hline Disk 1 & 0.29 & 12 & 5.2 \\
Disk 2 & 3100 & 0.038 & 0.021 \\
Disk 3 & 1.5 & 4.0 & 0.13 \\
\hline \hline
\end{tabular}

The least square fit for Eq. 7. The $a$ parameters were fitted so that when the model was integrated along the line of sight it give a prediction of brightness temperature for given $(l, b)$ direction. Hence the units of $a$ are $\mathrm{K} / \mathrm{kpc}$.
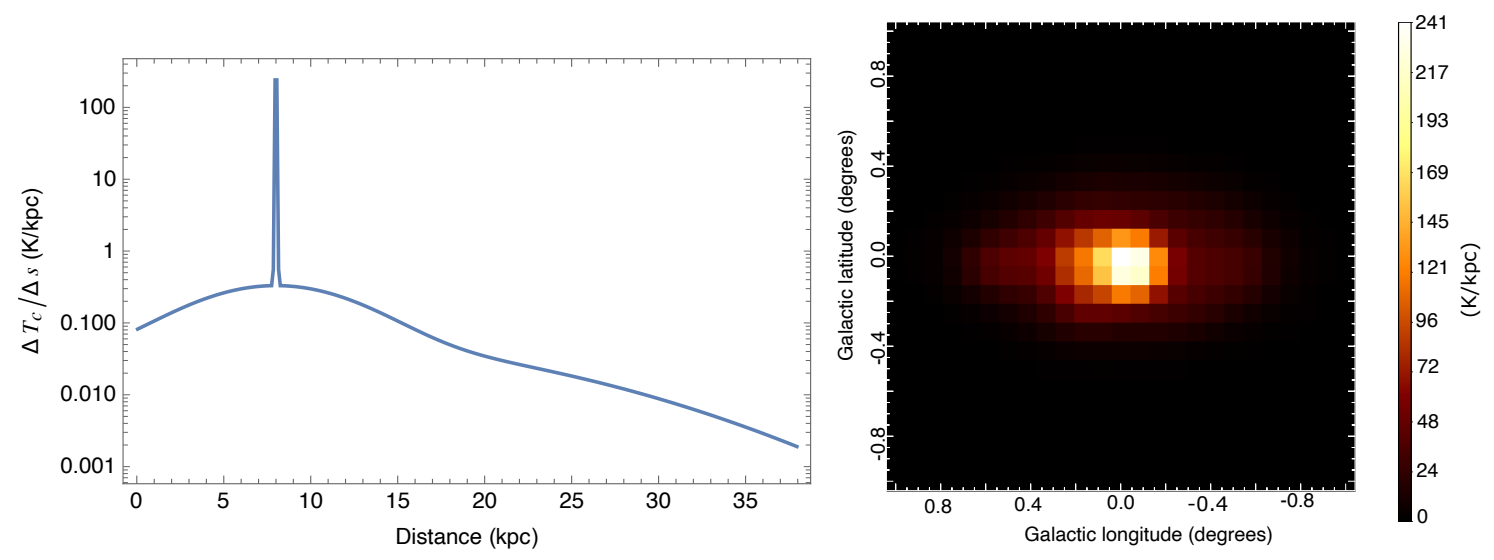

Figure 1: Continuum emission model. Left: Profile of $\Delta T_{c} / \Delta s$ for $(l, b)=\left(0^{\circ}, 0^{\circ}\right)$. Right: Cross section of $\Delta T_{c} / \Delta s$ at a distance of $8 \mathrm{kpc}$ from the solar system.

\subsection{Algorithm}

For each bin in velocity space in which the modulus of the signal exceeds $0.15 \mathrm{~K}$, we find the eight best-fitting distance solutions in distance bins of $50 \mathrm{pc}$. The signal is then distributed over those distance solutions using weights that are calculated as in Macias et al. [8].

As we ignore proper motion of gas clouds relative to the local average flow and the finite width of the signal from individual clouds, there is more signal without distance solution than with the deconvolution technique of Pohl et al. [11]. This signal is placed according to the distance solutions at the closest velocity covered in the gas-flow model, but the radiation transport is separately calculated for each velocity bin. Then the distance resolution is reduced by a factor two by combining neighboring distance bins. This fixes the line-of-sight distribution of the signal.

To determine the amplitude of the signal we create a set of 60 logarithmically spaced model signals with total $T_{l}$ ranging from $0.15 \mathrm{~K}$ to more than $10^{3} \mathrm{~K}$, for which we solve eq. 5 . We then search for the one model temperature that best matches the observed brightness. If the observed value is beyond the range of model values, we pick the closest one, otherwise we use linear interpolation between the two nearest models. The rms deviation between model and observed spectrum is computed and retained as accuracy parameter to the model file. Averaged over the area 

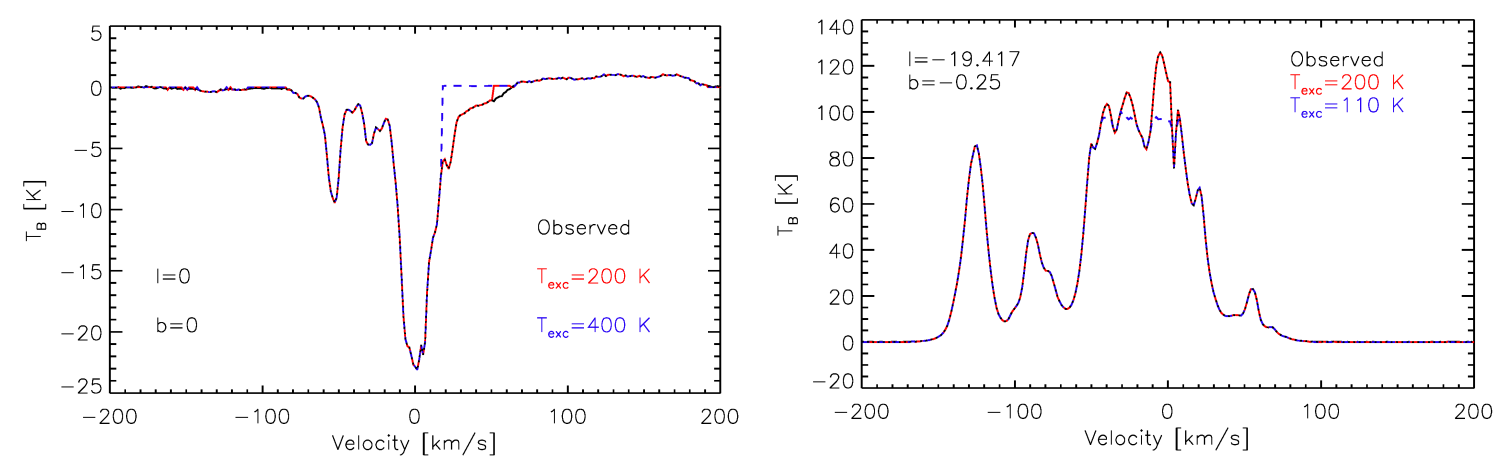

Figure 2: Comparison of the observed $\mathrm{HI}$ spectrum observed toward the Galactic Center (left panel) and a line of sight with a high intensity peak (right panel).

of interest, we see the smallest rms deviation with $T_{\mathrm{exc}}=200 \mathrm{~K}$.

Note that we place gas seen in absorption also on the far side, although it does not provide much absorption there because it is likely behind the continuum source. Otherwise we would construct a near-heavy Galaxy in regions of high absorption.

Figure 2 presents modelled and observed $\mathrm{H} I$ spectra for the line of sight toward the Galactic Center and an area with high intensity peaks in the line signal for two values of the excitation temperature, $T_{\mathrm{exc}}$. To be noted from the left panel of the figure is that solving the radiation transport equation with continuum emission can reproduce strong absorption features and provide an estimate of the $\mathrm{HI}$ column density where absorption occurs. Modelling negative line intensity becomes difficult for higher values of $T_{\mathrm{exc}}$, in particular for velocities for which the distance solutions are predominantly behind the region of high continuum emissivity. Whereas for $T_{\mathrm{exc}}=200 \mathrm{~K}$ that happens in only a narrow band around $v=50 \mathrm{~km} \mathrm{~s}^{-1}$, implying that only a small fraction of the gas is poorly modelled, a larger mismatch is seen for $T_{\mathrm{exc}}=400 \mathrm{~K}$. The $(l, b)=\left(0^{\circ}, 0^{\circ}\right)$ in the top panel is almost perfectly fit by $T_{\mathrm{exc}}=110 \mathrm{~K}$ but that excitation temperature doesn't works well for other lines of sight with high intensity peaks an instance of which is shown in the right panel.

In reality one should expect to find gas clouds on the line of sight that have different excitation temperatures. It is quite conceivable that the absorption feature around $v=50 \mathrm{~km} \mathrm{~s}^{-1}$ in the top panel of figure 2 is caused by relatively little cold gas immediately in front of the Galactic Center, whereas most of the gas clouds have temperatures of a few hundred Kelvin. We find it impossible to account for variable excitation temperature without introducing a large number of free parameters that are poorly, if at all, constrained. Hence the decision to use a uniform excitation temperature.

\section{Results}

The new HI templates where fit to the Fermi-LAT data in a similar way to as done in Macias et al. [9]. The likelihood for different excitation temperatures is shown in Fig. 3. 


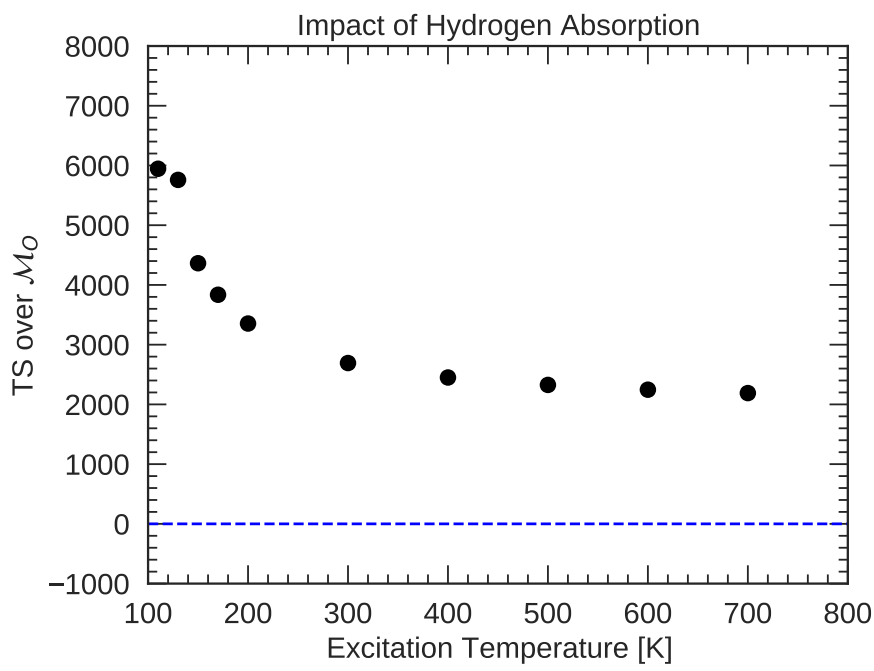

Figure 3: The $\mathrm{TS}=\log \left(\mathcal{L}_{i} / \mathcal{L}_{0}\right)$ in favor of a given excitation temperature hydrodynamic gas model over the old model $\left(\mathcal{M}_{O}\right)$ which doesn't account for the continuum emission. Note that $\mathcal{M}_{O}$ assumes the hydrodynamic gas maps proposed in [8] and has a likelihood $\mathcal{L}_{0}$ while an instance of the new model with excitation temperature $T_{i}$ has a likelihood $\mathcal{L}_{i}$.

As can be seen accounting for continuum emission improves the Fermi-LAT fit by $\Delta \log ($ likelihood $) ~$ 1000 .

In table 2 we show that the significance of a dark matter based template is below the $4 \sigma$ significance threshold once the expected unresolved millisecond pulsar population in the form of a nuclear bulge and boxy bulge are accounted for. Therefore, even though the new diffuse galactic background templates improve the fit to the Fermi-LAT data they do not change the conclusion that dark matter template is not required once the more standard astrophysical templates are accounted for.

Table 2: Log-likelihood values for the astrophysical background model and Galactic Center excess templates.

\begin{tabular}{lllllll}
\hline \hline Base & Source & $-\log \left(\mathcal{L}_{\text {Base }}\right)$ & $-\log \left(\mathcal{L}_{\text {Base+Source }}\right)$ & $\mathrm{TS}_{\text {Source }}$ & d.o.f & Significance \\
& & & & & \\
\hline Baseline+NB+BB & Cored ellipsoidal & -3262131.13 & -3262133.82 & 5.38 & 15 & $0.5 \sigma$ \\
Baseline+NB+BB & NFW & -3262131.13 & -3262131.31 & 0.37 & 15 & $0.0 \sigma$ \\
Baseline+NB+BB & NFW ellipsoidal & -3262131.13 & -3262131.98 & 1.71 & 15 & $0.1 \sigma$ \\
Baseline+NB+BB & Cored & -3262131.13 & -3262143.65 & 25.04 & 15 & $3.1 \sigma$ \\
\hline \hline
\end{tabular}

The baseline model is a combination of the new hydrodynamic gas maps introduced in this work (divided in for rings), inverse Compton maps computed with GALPROP V56 (divided in six rings), the 4FGL point sources [2], the Fermi Bubbles (FB) introduced in [9], Sun and Moon, isotropic and Loop I template (see Table I in [1]). Additional sources considered in the analysis are: Nuclear bulge (NB) [10], boxy bulge (BB) [5], NFW profile with $\gamma=1.2$, cored dark matter [12] and ellipsoidal versions of these two DM templates (see Fig. 3 in [1]). The maximized likelihoods $(\mathcal{L})$ are given for the Base and Base+Source models. The statistical significance for each new source is obtained by computing the $\mathrm{TS}_{\text {Source }}$ as shown in Eq. 2.5 of [9]. 


\section{References}

[1] Abazajian, K. N., Horiuchi, S., Kaplinghat, M., Keeley, R. E., \& Macias, O. 2020. https: //arxiv.org/abs/2003.10416

[2] Abdollahi, S., et al. 2019. https://arxiv.org/abs/1902.10045

[3] Bissantz, N., Englmaier, P., \& Gerhard, O. 2003, , 340, 949, doi: 10.1046/j .1365-8711. $2003.06358 . \mathrm{x}$

[4] Calabretta, M. R., Staveley-Smith, L., \& Barnes, D. G. 2014, , 31, e007, doi: 10 . 1017/pasa . 2013.36

[5] Coleman, B., Paterson, D., Gordon, C., Macias, O., \& Ploeg, H. 2020, Mon. Not. Roy. Astron. Soc., 495, 3350, doi: 10.1093/mnras/staa1281

[6] Draine, B. T. 2011, Physics of the Interstellar and Intergalactic Medium

[7] Jóhannesson, G., Porter, T. A., \& Moskalenko, I. V. 2018, , 856, 45, doi: 10 . 3847/1538-4357/ aab26e

[8] Macias, O., Gordon, C., Crocker, R. M., et al. 2018, Nature Astronomy, 2, 387, doi: 10.1038/ s41550-018-0414-3

[9] Macias, O., Horiuchi, S., Kaplinghat, M., et al. 2019. https: //arxiv . org/abs/1901.03822

[10] Nishiyama, S., Yasui, K., Nagata, T., et al. 2013, ApJ. Lett., 769, L28. http://stacks. iop.org/2041-8205/769/i=2/a=L28

[11] Pohl, M., Englmaier, P., \& Bissantz, N. 2008, , 677, 283, doi: 10. 1086/529004

[12] Read, J. I., Agertz, O., \& Collins, M. L. M. 2016, Mon. Not. Roy. Astron. Soc., 459, 2573, doi: $10.1093 / \mathrm{mnras} / \mathrm{stw} 713$

[13] Reich, P., Testori, J. C., \& Reich, W. 2001, , 376, 861, doi: 10 . 1051/0004-6361:20011000

[14] Testori, J. C., Reich, P., Bava, J. A., et al. 2001, , 368, 1123, doi: 10.1051/0004-6361: 20010088 